\title{
Protective Effects of Oridonin on Acute Liver Injury via Impeding Posttranslational Modifications of Interleukin-1 Receptor-Associated Kinase 4 (IRAK4) in the Toll-Like Receptor 4 (TLR4) Signaling Pathway
}

\author{
Min Shi $\mathbb{D}^{1},{ }^{1}$ Yilin Deng, ${ }^{2}$ Heguo Yu $\left(\mathbb{D},{ }^{3}\right.$ Ling Xu $\mathbb{D}^{4},{ }^{4}$ Cuicui Shi, ${ }^{5}$ Jiong Chen, 4 \\ Guangming Li $\mathbb{D},^{5}$ Yiqi Du $\mathbb{D},{ }^{1}$ and Yu-gang Wang $\mathbb{D}^{4}$ \\ ${ }^{1}$ Department of Gastroenterology, Changhai Hospital, Second Military Medical University/Naval Medical University, \\ Shanghai 200433, China \\ ${ }^{2}$ Department of Gastrointestinal Cancer Biology, Tianjin Medical University Cancer Institute and Hospital, National Clinical \\ Research Center for Cancer, Key Laboratory of Cancer Prevention and Therapy, Tianjin 300060, China \\ ${ }^{3}$ NPFPC Key Laboratory of Contraceptives and Devices, Shanghai Institute of Planned Parenthood Research (SIPPR), Institutes of \\ Reproduction and Development, Fudan University, Shanghai 200000, China \\ ${ }^{4}$ Department of Gastroenterology, Shanghai Tongren Hospital, Shanghai Jiao Tong University School of Medicine, \\ Shanghai 200336, China \\ ${ }^{5}$ Department of Gastroenterology, Xinhua Hospital, Shanghai Jiao Tong University School of Medicine, Shanghai 200092, China
}

Correspondence should be addressed to Yiqi Du; duyiqi@hotmail.com and Yu-gang Wang; wyg0061@shtrhospital.com

Received 1 June 2019; Accepted 9 August 2019; Published 12 September 2019

Academic Editor: Oleh Andrukhov

Copyright ( 2019 Min Shi et al. This is an open access article distributed under the Creative Commons Attribution License, which permits unrestricted use, distribution, and reproduction in any medium, provided the original work is properly cited.

Objective. Recent researches have demonstrated that inflammation-related diseases are effectively regulated by posttranslational modifications (PTMs) including phosphorylation and acetylation. Our previous study found a new acetyltransferase inhibitor, oridonin, which had a protective effect on acute liver injury (ALI). In the present study, we further investigated its protective mechanism against D-galactosamine (D-Gal) combined with lipopolysaccharide- (LPS-) induced ALI in mice. Methods. Intraperitoneal injections of LPS (40 $\mu \mathrm{g} /$ mouse)/D-Gal $(5 \mathrm{mg} / \mathrm{mouse})$ were given to the mice, and the experimental group was pretreated with intraperitoneal injection of oridonin $(0.2 \mathrm{mg} / \mathrm{mouse})$. To elucidate the protective mechanism of oridonin, we collected liver specimens and used RNA-sequencing (RNA-Seq) analysis. We focused on the genes that were upregulated by LPS/D-Gal and downregulated after pretreatment with oridonin. The downregulated genes examined by Gene Ontology (GO) and Kyoto Encyclopedia of Genes and Genomes (KEGG) pathway analysis were further verified by real-time polymerase chain reaction (PCR) and western blot. Results. GO analysis showed that genes that were downregulated after pretreatment with oridonin were extremely concentrated in immune response, chemotaxis, and inflammatory response. Real-time PCR confirmed that the expression of these genes was upregulated by LPS/D-Gal induction and reduced after treatment with oridonin, which was consistent with RNA-Seq results. KEGG pathway analysis showed a significantly enriched downregulated gene that was present in the Toll-like receptor (TLR) 4 signaling cascade. Our results manifested that phosphorylation levels of upstream signaling molecules in the TLR4 signaling cascade, including extracellular signal-regulated kinase (ERK), P38, and I $\kappa$ B, were significantly inhibited by oridonin. Furthermore, LPS/D-Gal stimulation triggered posttranslational modifications of related gene loci in the TLR4 signaling pathway, including phosphorylation of IL-1 receptor-associated kinase 4 (IRAK4 T345/S346) and acetylation of IRAK4 (K34). However, after treatment with oridonin, the modification pattern of IRAK4 expression stimulated by LPS/D-Gal was suggestively attenuated. Conclusion. Our study revealed that the protective effects of oridonin on LPS/D-Gal-induced ALI mediated by inhibition of the PTMs of IRAK4, including phosphorylation of T345/S346 and acetylation of K34. 


\section{Introduction}

Severe acute liver injury (ALI) is a rapid pathological process that can be life-threatening [1]. Hepatitis virus, adverse drug effects, alcohol abuse, metabolic syndrome, and other factors can lead to ALI. ALI is a common starting point in a series of liver diseases that can result in acute or chronic liver failure, cirrhosis, and hepatocellular carcinoma [2]. Because these liver diseases account for around 2 million deaths per year globally, the global burden of liver disease is anticipated to rise; therefore, therapies for ALI are urgently needed $[3,4]$.

More and more studies have found that posttranslational modifications (PTMs), for example, acetylation, methylation, phosphorylation, ubiquitination, and SUMOYlation, can control protein activity, facilitate protein interactions, and change protein subcellular localization, leading to regulation of important signaling pathways [5-7]. PTMs have a critical role in the duration and extent of the inflammatory response in liver injury through targeting the signaling molecules $[3,8,9]$. Interpretation of the correlation between various protein modifications is, to some extent, one of the keys to elucidate the mechanisms of liver injury $[4,10]$. Regulation of PTMs might act as a significant therapeutic target during the liver injury treatment [11, 12]. Furthermore, related efficient inhibitors are now being explored as novel directions in drug research $[13,14]$.

Oridonin is a well-known diterpenoid acquired from the Chinese medicinal herb Rabdosia rubescens, also recognized as Dong Ling Cao [15]. Over the last few decades, oridonin has gained widespread attention because of its complex role in cancer treatment, such as leukemia $[16,17]$, lymphogenous malignancy [18], hepatocellular carcinoma [19], and colorectal cancer [20]. However, it has been documented that oridonin has many other therapeutic effects, comprising anti-inflammation [21, 22], neuroprotection [23], and antihepatic fibrosis [24]. Our previous study demonstrated that oridonin had protective effects on ALI stimulated by LPS/D-Gal, manifested as an improved survival rate, ameliorated histological abnormalities, and impaired liver function [25]. Additionally, we discovered that oridonin acted as an inhibitor of several acetyltransferases, such as pCAF, P300, Tip60, and GCN5 [26]. Therefore, in the current study, we intended to demonstrate whether the underlying protection mechanism of oridonin in ALI was related to the regulation of PTMs.

\section{Materials and Methods}

2.1. Reagents. Oridonin ( $>98 \%$ purity; $\mathrm{C}_{20} \mathrm{H}_{28} \mathrm{O}_{6} ; \mathrm{M} . \mathrm{W}$. 360.42), obtained from Selleck (USA), was suspended in dimethyl sulfoxide, stored at $-20^{\circ} \mathrm{C}$, and thawed prior to use. Lipopolysaccharide (LPS) (Escherichia coli, 0111:B4), D-Gal, and thiol-specific antioxidant (TSA) were purchased from Sigma (USA). A myeloperoxidase (MPO) assay kit was from Nanjing Jiancheng Bioengineering Institute (China). A ReverTra Ace qPCR RT Kit was purchased from Toyobo (Japan). A SYBR Green Real-time PCR Master Mix was acquired from ExCell Bio (China). RIPA lysis buffer was purchased from KeyGen Biotech
(China). Antibodies against ERK, P-ERK, P38, P-P38, $\mathrm{I} \kappa \mathrm{B}, \mathrm{P}-\mathrm{I} \kappa \mathrm{B}$, interleukin-1 receptor-associated kinase 4 (IRAK4), P-IRAK4 (T345/S346), histone 3 (H3), H4, $\alpha$ tubulin, acetyl- $\alpha$-tubulin (K40), and GAPDH were obtained from Cell Signaling Technology (USA). Pan-anti-acetyl-lysine monoclonal antibodies were generated by our own laboratory, as described previously [27]. Horseradish peroxidase(HRP-) conjugated goat anti-mouse and goat anti-rabbit antibodies were from Beyotime (China).

2.2. Generation of Peptides and Anti-Acetyl-IRAK4 (K34) Monoclonal Antibodies. Synthesized peptide sequence of acetyl-IRAK4 was PQEGWKK (ac) LAVAIK. Peptides and anti-acetyl-lysine-IRAK4 (K34) monoclonal antibodies were manufactured by GL Biochem (Shanghai, China).

2.3. Cell Culture and Assay of Acetyltransferase Inhibitory Activity of Oridonin. The mouse immortalized stellate cell line JS1 was acquired from Cell Resource Center (Shanghai Institutes for Biological Sciences, China). These cells were grown in Dulbecco's modified Eagle's medium with $10 \%$ fetal bovine serum and $1 \%$ penicillin/streptomycin at a $37^{\circ} \mathrm{C}$ incubator with $5 \% \mathrm{CO}_{2}$. JS1 cells were seeded in $10 \mathrm{~cm}$ dishes and incubated in culture medium for $24 \mathrm{~h}$. The cells were pretreated with oridonin $(5,10$, and $15 \mu \mathrm{m})$ for $3 \mathrm{~h}$ before adding TSA $(1 \mathrm{mM})$. The cells were harvested after $4 \mathrm{~h}$ of TSA treatment. We performed western blot to detect the level of $\mathrm{H} 3, \mathrm{H} 4$, acetylated histone $\mathrm{H} 3$, acetylated histone $\mathrm{H} 4, \alpha$-tubulin, and acetylated $\alpha$ tubulin and compared with GAPDH as a control.

2.4. Animals. Animals were handled as per the institutional animal care policies of the Shanghai Institute of Planned Parenthood Research. Female C57BL/6 mice (specific pathogen-free, 20-22 g, wild type) were obtained from SIPPR-BK Animal Co. Ltd. (Shanghai, China). The mice were given with a regular chow diet and water. They were accommodated under standard laboratory environments $\left(21 \pm 2^{\circ} \mathrm{C}\right.$, $12 \mathrm{~h}$ light/dark cycle) and were adapted for a minimum of 2 weeks before beginning any experiment.

2.5. Experimental Design. ALI was prompted in mice with intraperitoneal injection of LPS $(40 \mu \mathrm{g} / 0.5 \mathrm{~mL})$ combined with D-Gal $(5 \mathrm{mg} / 0.5 \mathrm{~mL})$. Animals were randomly separated into five groups $(n=6$ each): control group (a), ALI group (b), two oridonin treatment groups (c and d), and oridonin control group (e). In group c, oridonin $(0.2 \mathrm{mg} / 0.5 \mathrm{~mL})$ was given $1 \mathrm{~h}$ prior to LPS/D-Gal challenge, and in group $\mathrm{d}$, oridonin $(0.2 \mathrm{mg} / 0.5 \mathrm{~mL})$ was given every $4 \mathrm{~d}$ for a total of three doses, where the final dose was given $1 \mathrm{~h}$ before LPS/D-Gal challenge. All animals were killed by amputation at $6 \mathrm{~h}$ following LPS/D-Gal challenge. The liver tissues were acquired and preserved at $-80^{\circ} \mathrm{C}$ for future use.

2.6. Transcriptome Analysis of Gene Expression Profiles of ALI upon Oridonin Treatment and Validation of the Differentially Expressed Genes (DEGs) by Real-Time Quantitative Polymerase Chain Reaction ( $q P C R$ ). The total mRNAs of five groups were isolated and analyzed using next-generation RNA-sequencing (RNA-Seq) technology on an Illumina 
TABLE 1: Sequence of primers used for qPCR.

\begin{tabular}{lcc}
\hline Target genes & \multicolumn{1}{c}{ Forward primers } & Reverse primers $^{\prime}$ \\
\hline IL-1 $\alpha$ & $5^{\prime}$-AGT ATC AGC AAC GTC AAG CAA-3 & $5^{\prime}$-TCC AGA TCA TGG GTT ATG GAC TG-3 \\
IL-1 $\beta$ & $5^{\prime}$-GAA ATG CCA CCT TTT GAC AGT G-3' & $5^{\prime}$-TGG ATG CTC TCA TCA GGA CAG-3' \\
IL-6 & $5^{\prime}$-TAG TCC TTC CTA CCC CAA TTT CC-3' & $5^{\prime}$-TTG GTC CTT AGC CAC TCC TTC-3' \\
TNF- $\alpha$ & $5^{\prime}$-CAG GCG GTG CCT ATG TCT C-3' & $5^{\prime}$-CGA TCA CCC CGA AGT TCA GTA G-3' \\
CCL2 & $5^{\prime}$-TAA AAA CCT GGA TCG GAA CCA AA-3' & $5^{\prime}$-GCA TTA GCT TCA GAT TTA CGG GT-3' \\
CCL3 & $5^{\prime}$-TGT ACC ATG ACA CTC TGC AAC-3' & $5^{\prime}$-CAA CGA TGA ATT GGC GTG GAA-3' \\
CCL4 & $5^{\prime}$-TTC CTG CTG TTT CTC TTA CAC CT-3' & $5^{\prime}$-CTG TCT GCC TCT TTT GGT CAG-3' \\
CCL5 & $5^{\prime}$-GTG CCC ACG TCA AGG AGT AT-3' & $5^{\prime}$-GGG AAG CTA TAC AGG GTC A-3' \\
CCL7 & $5^{\prime}$-CCA CAT GCT GCT ATG TCA AGA-3' & $5^{\prime}$-ACA CCG ACT ACT GGT GAT CCT-3' \\
CXCL1 & $5^{\prime}$-ACT GCA CCC AAA CCG AAG TC-3' & $5^{\prime}$-TGG GGA CAC CTT TTA GCA TCT T-3' \\
CXCL10 & $5^{\prime}$-CCA AGT GCT GCC GTC ATT TTC-3' & $5^{\prime}$-TCC CTA TGG CCC TCA TTC TCA-3' \\
GAPDH & $5^{\prime}$-TCC AAG GAG TAA GAA ACC CTG GAC-3' & $5^{\prime}$-GTT ATT ATG GGG GTC TGG GAT GG-3' \\
\hline
\end{tabular}

HiSeq 2000 platform (Genenergy Bio, Shanghai, China) to outline their global gene expression patterns. For qPCR verification, total RNA was collected from five groups with or without oridonin treatment. Fluorescent qPCR was carried out on an ABI 7500 Fast Real-Time PCR detective system (Applied Biosystems, CA, USA) for chemokines and inflammatory cytokines. The SYBR Green qPCR system was $20 \mu \mathrm{L}$ in total, which consisted of $10 \mathrm{~mL}$ SYBR Premix Ex Taq, $1.6 \mathrm{~mL}$ of the forward and reverse primers, $7 \mathrm{~mL}$ of doubledistilled water, and $1.4 \mathrm{~mL}$ cDNA template. The reaction conditions were performed according to the supplier's instructions. GAPDH served as a control. All samples were run in triplicate. The relative expression was calculated by the $2^{-\Delta \Delta \mathrm{Ct}}$ method. Table 1 shows the list of primers.

2.7. Hepatic Myeloperoxidase (MPO) Activity Assay. Activity of MPO was measured via spectrophotometry using the MPO assay kit.

2.8. Protein Isolation and Immunoblot. RIPA lysis buffer with protease inhibitor cocktail was used to extract the total protein. Protein concentrations were measured via the BCA method. Equivalent quantities of protein were utilized to examine ERK, P-ERK, P38, P-P38, IRAK4, P-IRAK4 (S345/T346) and acetyl-IRAK4 (K34), I $\kappa$ B, P-I $\kappa B, H 3, H 4$, $\alpha$-tubulin, acetyl- $\alpha$-tubulin (K40), and pan-acetylation in comparison with GAPDH as a control. Goat anti-rabbit or goat anti-mouse IgG-HRP antibody was the secondary antibody. An enhanced chemiluminescence (ECL) detection kit was utilized to visualize the protein bands.

2.9. Statistical Analysis. All results are showed as the mean \pm SD and from a minimum of three different independent experiments. Student's $t$-test was utilized to determine the difference between two groups. A $\chi^{2}$ test was utilized to the evaluation of sample rates among multiple samples. All analyses were accomplished with GraphPad Prism 6. $P<0.05$ and $P<0.01$ signified statistical significance.

\section{Results}

3.1. Oridonin Decreased Acetylation of $\mathrm{H} 3, \mathrm{H} 4$, and $\alpha$ Tubulin in JS1 Cells and LPS/D-Gal-Induced ALI. To assess the acetyltransferase inhibitory activity of oridonin in vitro and in vivo, we studied manifestation of acetyl- $\alpha$-tubulin (K40), acetyl-H3, and acetyl-H4. The results indicated that the acetylation level of tubulin (K40), $\mathrm{H} 3$, and $\mathrm{H} 4$ decreased suggestively when the final concentration of oridonin was $15 \mu \mathrm{m}$ (Figure 1(a)). There was a noteworthy rise in the overall pan-acetylation level, especially the expression of acetyl$\mathrm{H} 3$, acetyl-H4, and acetyl- $\alpha$-tubulin in LPS/D-Gal-induced ALI. However, the overall pan-acetylation level reduced in a concentration-dependent way upon oridonin treatment (Figure 1(b)). Our study established that oridonin harbors the activity of acetyltransferase inhibitor in vitro and in vivo.

3.2. Bioinformatics Revealed the GOs and Signaling Pathways Regulated by Oridonin. We focused on the genes that were induced by LPS/D-Gal compared with the control group and then downregulated by oridonin treatment. Screening analysis indicated that LPS/D-Gal stimulated expression of 581 genes. In the oridonin-treated group c, 121 genes with fold changes $\geq 2$ were downregulated by oridonin (Supplementary Table S1). GO analysis suggested that the downregulated genes were extremely enriched in chemotaxis, locomotor activity, inflammatory response, and immune response. KEGG pathway analysis presented abundance of downregulated genes in several cascades, comprising the NOD-like receptor signaling pathway, mitogen-activated protein kinase (MAPK) signaling pathway, and TLR signaling pathway (Supplementary Figure S1). In the oridonin-treated group d, 278 genes with fold changes $\geq 2$ were downregulated by three doses of oridonin (Supplementary Table S2). GO analysis showed that the downregulated genes were greatly enriched in immune response, chemotaxis, and inflammatory response. KEGG pathway analysis indicated enhancement of 


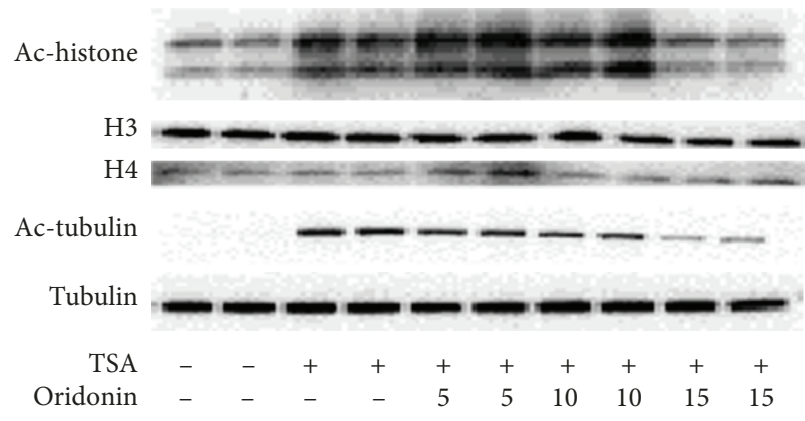

(a)

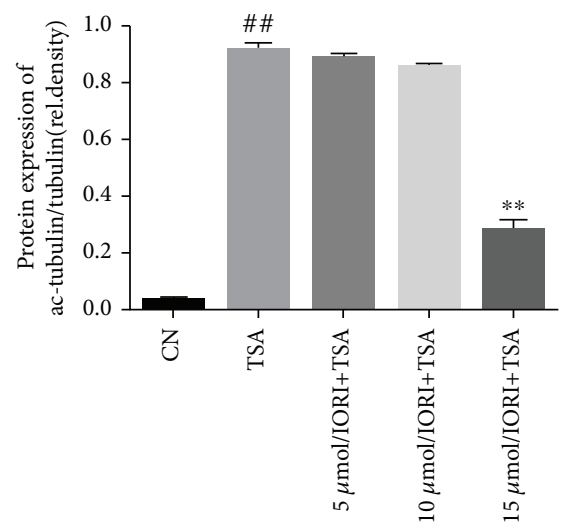

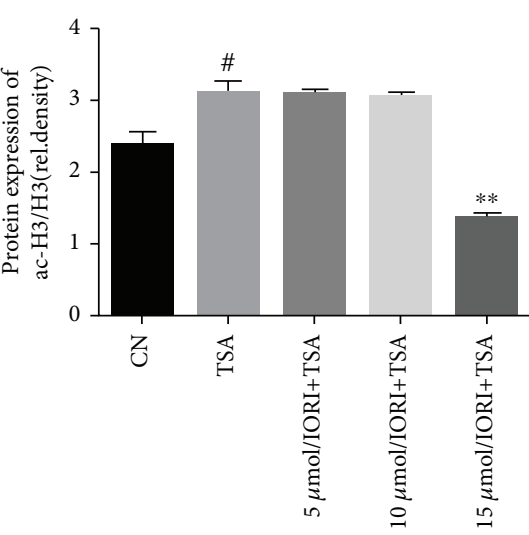

(c)

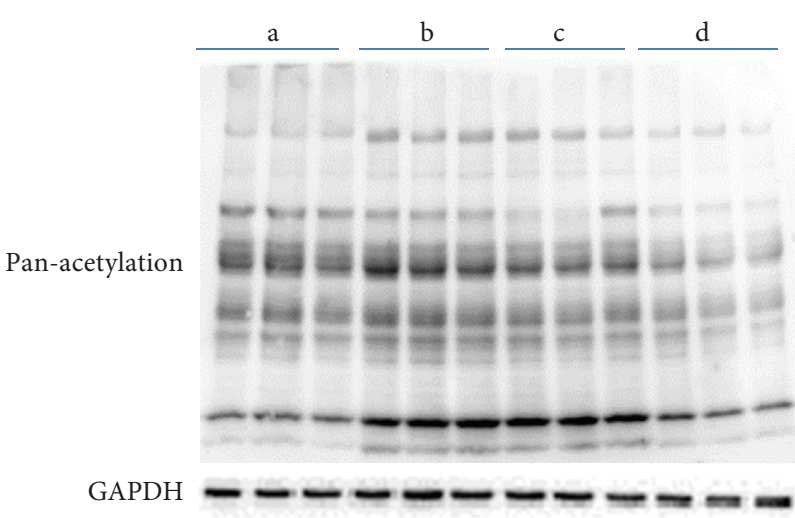

(b)

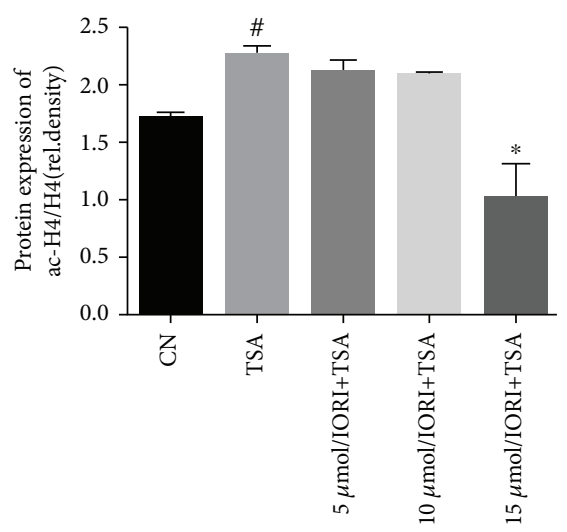

FIgURE 1: Acetyltransferase inhibitory activity of oridonin was assessed in vivo and in vitro. (a) JS1 cells were seeded in $10 \mathrm{~cm}$ dishes and incubated for $24 \mathrm{~h}$. The cells were pretreated with oridonin $(5,10$, or $15 \mu \mathrm{m})$ for $3 \mathrm{~h}$ before adding $1 \mathrm{mM}$ TSA. The cells were harvested after $4 \mathrm{~h}$ of TSA treatment. Western blot showed that oridonin decreased acetylation of $\mathrm{H} 3, \mathrm{H} 4$, and $\alpha$-tubulin induced by TSA. The inhibitory effect of oridonin was obvious when the concentration was $15 \mu \mathrm{m}$. (b) There was a substantial rise in the overall pan-acetylation level, especially expression of acetyl-H3, acetyl-H4, and acetyl- $\alpha$-tubulin in LPS/D-Gal-induced ALI. The overall pan-acetylation level reduced in a concentration-dependent way upon oridonin treatment. (c) Band intensity in (a) was quantified by ImageJ. ${ }^{\#} P<0.05$, ${ }^{\# \#} P<0.01$ vs. control group; ${ }^{*} P<0.05,{ }^{* *} P<0.01$ vs. TSA treatment group.

downregulated genes in several cascades such as TLR and NOD-like receptor signaling pathways. Our previous research revealed that the prophylactic effects of oridonin were more obvious in group $\mathrm{d}$, so we used the bioinformatics interpretation of group $\mathrm{d}$, which better suggested the target of oridonin (Figure 2).

\subsection{Significantly Enriched GO Term-Related Genes Verified} by Real-Time PCR. To verify the outcomes of RNA-Seq, the genes involved in chemotaxis, immune, and inflammatory responses were subjected to real-time PCR. Hepatic expression of 11 chemotaxis and immune and inflammatory response-related genes (IL- $1 \alpha$, IL- $1 \beta$, IL- 6 , TNF- $\alpha$, CCL2, CCL3, CCL4, CCL5, CCL7, CXCL1, and CXCL10) was analyzed among the five groups. Coherent with RNA-Seq outcomes, expression of these 11 genes was elevated in the LPS/D-Gal group and reduced due to oridonin treatment. Oridonin suppressed chemotaxis, immune, and inflammatory response-related genes in a concentration-dependent way. These outcomes provide more evidence for the reliabil- ity of bioinformatics analysis, indicating that oridonin suppressed the production of cytokines involved in the immune and inflammatory processes, which might protect the liver from injury (Figure 3).

3.4. Oridonin Suppressed MPO Activity Prompted by $L P S / D-G a l$. Inflammatory response, including excessive inflammation-related cell activation and infiltration into liver tissues, contributes to LPS/D-Gal-induced liver injury [28]. A large amount of neutrophil infiltration was observed in an ALI model, characterized by high levels of MPO activity. Pretreatment with oridonin inhibited neutrophil infiltration into liver tissues as demonstrated by reduced MPO activity (Figure 4).

3.5. Participation of NF- $\kappa B$ and MAPK Signaling Pathways in the Anti-Inflammatory Impact of Oridonin on LPS/D-GalInduced ALI. KEGG analysis specified that the TLR4 signaling pathway was significantly enriched (Figure $2(\mathrm{c})$ ). NF- $\kappa \mathrm{B}$ and MAPK are two critical downstream cascades 


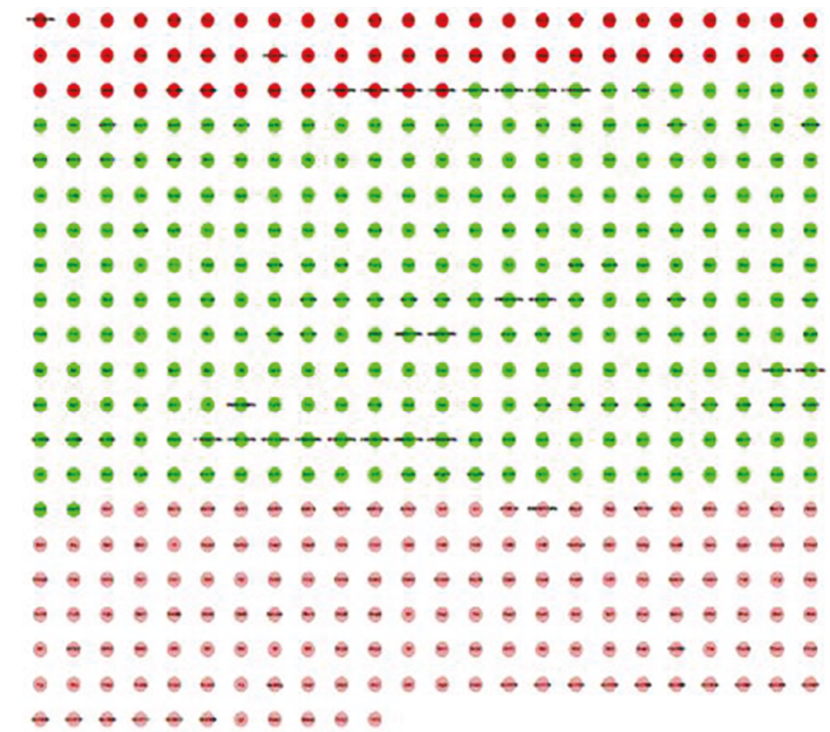

(a)

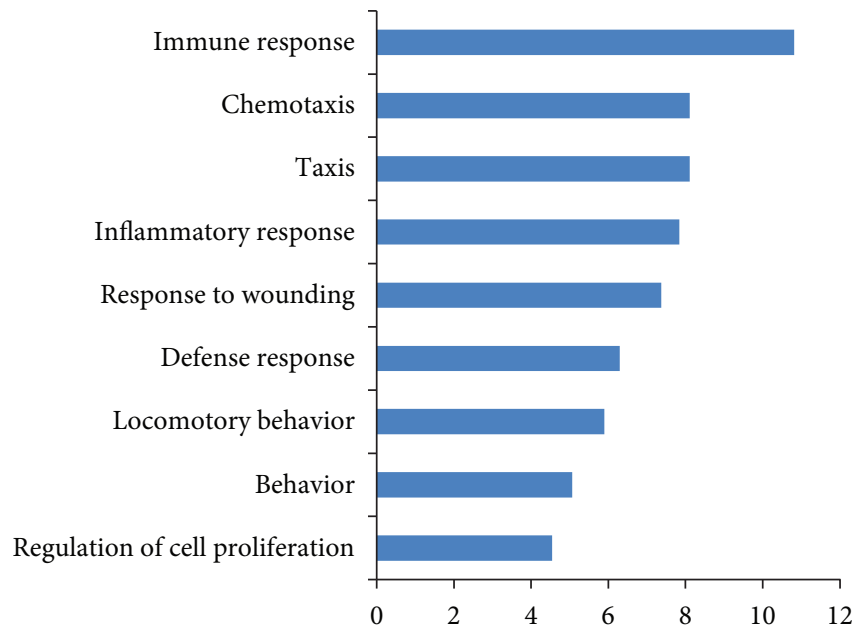

(b)

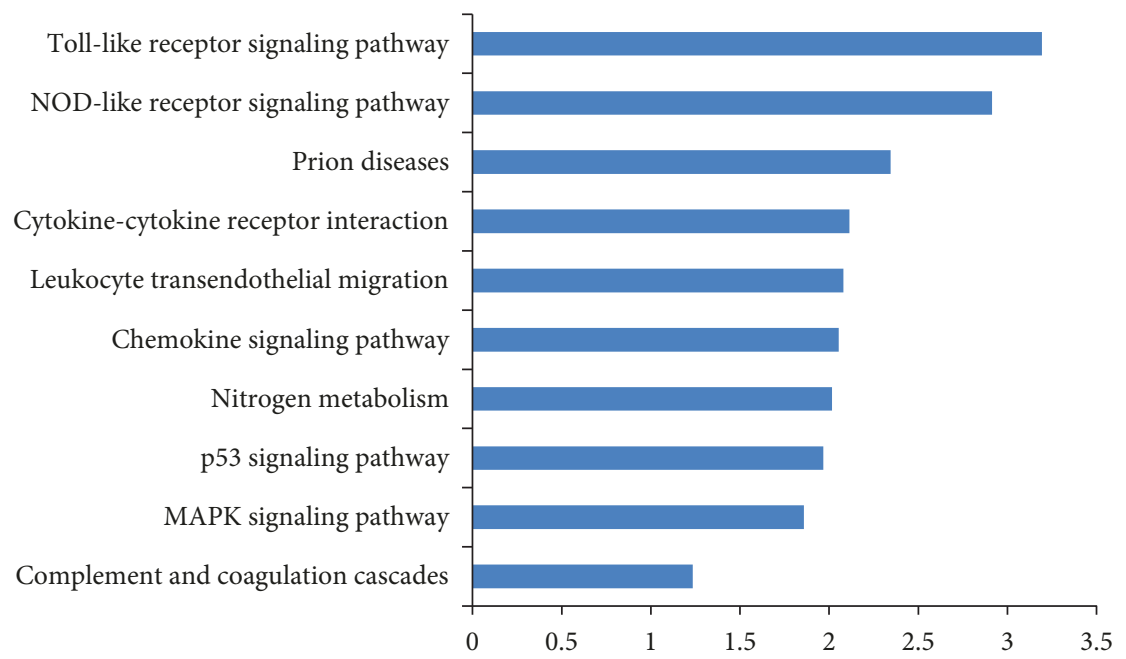

(c)

Figure 2: Continued. 


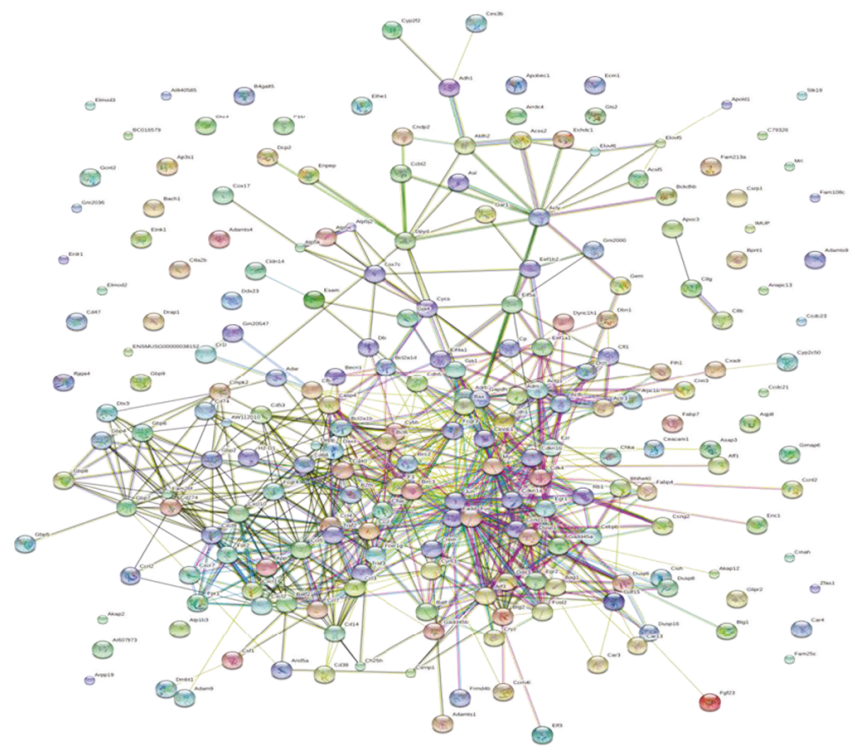

(d)

FIGURE 2: Effects of oridonin on gene expression profiles in LPS/D-Gal-induced ALI assayed by RNA-Seq. (a) Clustering analysis of gene expression profiles prompted by LPS/D-Gal challenge using Cytoscape software. The green part represents the genes that were upregulated by LPS/D-Gal. We focused on the target genes stimulated by LPS/D-Gal and downregulated by oridonin treatment in group d. The target genes were subjected to bioinformatics analysis. (b) GO analysis of target genes for biological processes revealed that downregulated genes were greatly enriched in immune response, chemotaxis, and inflammatory response. (c) KEGG pathway analysis of target genes showed that downregulated genes were enriched in several pathways including TLR and NOD-like receptor signaling pathways. (d) Interactions of the target genes demonstrated by KEGG pathway analysis.
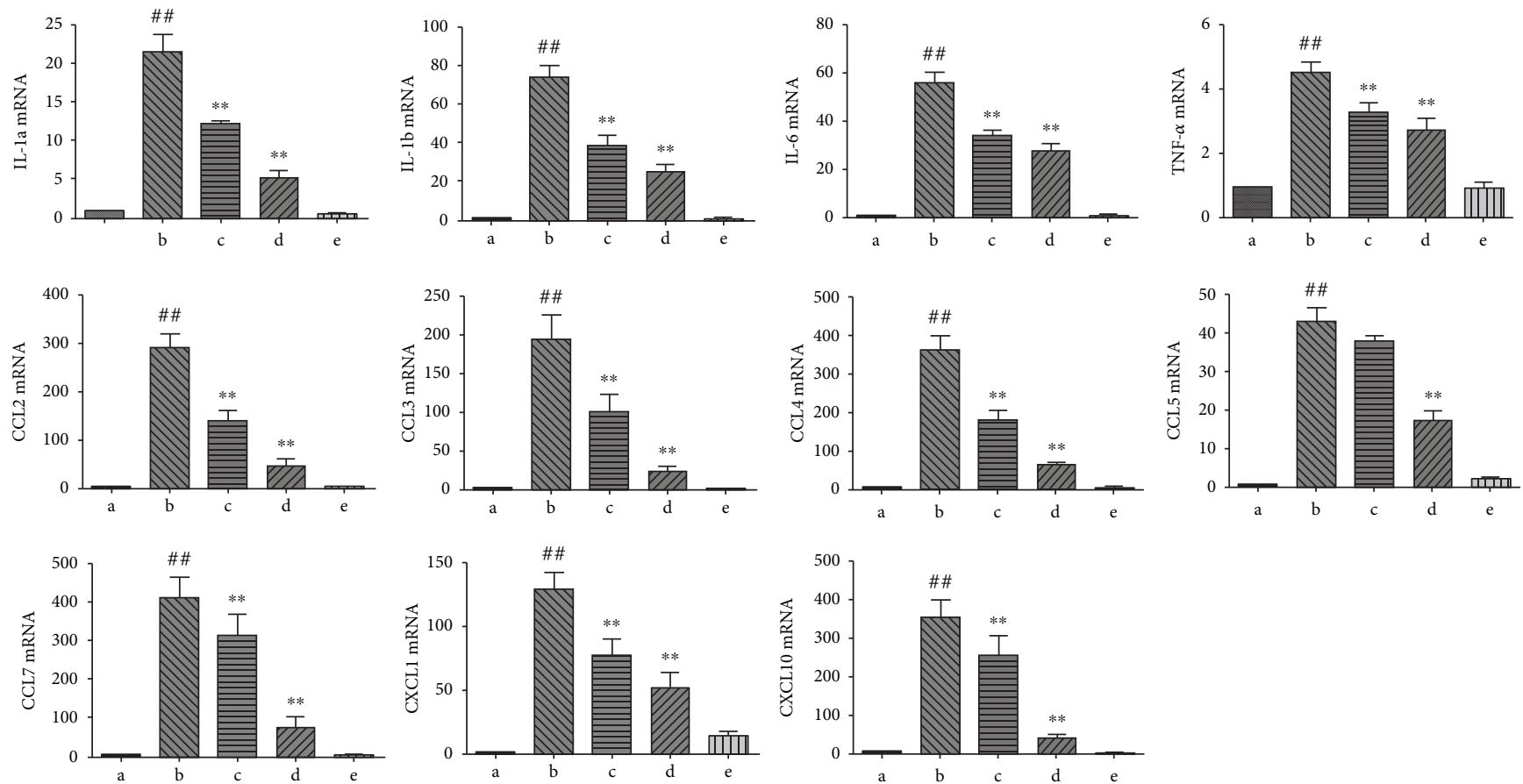

FIGURE 3: Significantly enriched GO term-related genes were validated by real-time PCR. Constant with RNA-Seq results, expression of GO term-related genes was elevated in the LPS/D-Gal group and decreased in a concentration-dependent way by oridonin treatment. ${ }^{\# \#} P<0.01$ vs. control group (a); ${ }^{* *} P<0.01$ vs. model group (b).

participating in LPS-stimulated TLR4 signal transduction [29]. Stimulation of the MAPK pathway causes initiation of transcription factor AP-1, which plays a critical role in the regulation of proinflammatory cytokines [30]. We established that the elevated levels of P-ERK and P-P38 prompted by LPS/D-Gal were abolished by pretreatment of mice with 


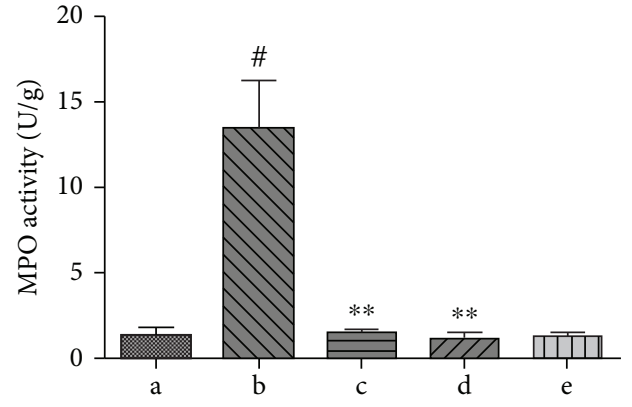

FIGURE 4: Oridonin suppressed MPO activity prompted by LPS/DGal. ${ }^{\#} P<0.05$ vs. control group (a); ${ }^{* *} P<0.01$ vs. model group (b).

oridonin. NF- $\kappa \mathrm{B}$ also has a vital role in controlling downstream signaling events that mediate proinflammatory gene transcription. In resting cells, NF- $\kappa \mathrm{B}$ is preserved in the cytoplasm by $\mathrm{I} \kappa \mathrm{B}$ inhibitors, which are phosphorylated and then quickly degraded due to stimuli, resulting in NF- $\kappa$ B nuclear activation [31]. It means that we could use the phosphorylation of $\mathrm{I} \kappa \mathrm{B}$ to indicate the activation of NF- $\kappa \mathrm{B}$. Thus, we analyzed the phosphorylation of $\mathrm{I} \kappa \mathrm{B}$ to further elucidate the anti-inflammatory mechanism of oridonin. As expected, phosphorylation of $\mathrm{I} \kappa \mathrm{B}$ was depressed by oridonin in a concentration-dependent way (Figure 5).

3.6. Modification (Phosphorylation and Acetylation) of IRAK4 Was Inhibited in LPS/D-Gal-Induced ALI by Oridonin. Protein PTMs have a deep impact on protein activity and stability along with binding capacity [32]. IRAK4 is indispensable for the MyD88-dependent TLR4 signaling pathway, including the activation of MAPK and NF- $\kappa$ B signaling cascades [33]. Initial studies revealed that the kinase activity of IRAK4 is required for the proper activation of MAPK and NF- $\kappa \mathrm{B}$ [34]. Furthermore, another study using LC-MS/MS analysis recognized three phosphorylation sites (T342, T345, and S346) within the stimulation loop, which are critical to the kinase activity of IRAK4 [35]. Our results indicated that oridonin inhibited expression of P-IRAK4 (T345/S346) induced by LPS/D-Gal. IRAK4 is presumed to interact with MyD88 through the death domain, and the downstream signaling pathway is blocked without their interaction [36]. UniProt database indicated that the K34 site that resides in the death domain could be acetylated. The acetylation status of IRAK4 (K34) may influence the interaction between IRAK4 and MyD88, resulting in the blockade of signal transduction. Thus, we further identified that oridonin inhibited the acetylation level of IRAK4 (K34) stimulated by LPS/D-Gal. All the results suggested that LPS/D-Gal-induced PTMs of IRAK4 and oridonin pretreatment reversed the changes. The impediment of PTMs of IRAK4 may be one of the reasons of the anti-inflammatory mechanisms of oridonin (Figure 6).

\section{Discussion}

An established model for studying ALI in mice involves coadministration of LPS plus D-Gal, which results in a pathological progression parallel to clinical ALI and is also an imperative platform for screening novel medications for the treatment of ALI [37]. Our previous researches have shown that oridonin improves the survival rate of mice and liver histopathology and reduces plasma alanine and aspartate aminotransferase levels, indicating that oridonin has significant protective effects on ALI [25].

We further explored the possible mechanisms underlying oridonin-mediated hepatoprotection. Transcriptome analysis indicated that the protective mechanism of oridonin might be correlated with suppression of TLR4-mediated immune and inflammatory responses. The MPO activity of the liver, which is believed to be a key step in the initiation of the inflammatory response, was markedly reduced by oridonin treatment. The parallel inhibition of chemotaxis and immune as well as inflammatory response-related genes' mRNA expression implies that obstruction of transcription might have a crucial role in the protective impact of oridonin. The production of inflammatory mediators is known to depend mainly on activation of transcription factor NF- $\kappa \mathrm{B}$ [38]. The crucial step in NF- $\kappa \mathrm{B}$ stimulation is $\mathrm{I} \kappa \mathrm{B}$ phosphorylation by IKK, and then, NF- $\kappa \mathrm{B}$ enters the nucleus and controls manifestation of inflammatory cytokines [38]. Activation of the MAPK family has a crucial function in a large number of cellular processes, including proliferation, differentiation, and expression of proinflammatory factors [39]. Our results revealed that oridonin inhibited the stimulation of NF- $\kappa \mathrm{B}$. The inhibitory effect was demonstrated by the reduction of $\mathrm{I} \kappa \mathrm{B}$ phosphorylation. At the same time, activation of the MAPK family members was inhibited by oridonin treatment. These results presented that the antiinflammatory impact of oridonin is partly dependent on the MAPK and NF- $\kappa \mathrm{B}$ signaling pathways, which indicates that the target of oridonin might be stimulated earlier due to LPS/D-Gal challenge.

TLR4 signaling originally causes congregation of the LPS/TLR4 recognition complex, which prompts a cascade of PTMs of proteins, for example, IRAK4. The crucial involvement of IRAK4 in TLR4-mediated inflammatory responses has been documented in knockout $(\mathrm{KO})$ studies [35]. Be in composition to IRAK1 KO mice, IRAK4 KO mice were absolutely unaffected to LPS-induced septic shock and did not have a cytokine response when challenged with numerous TLR ligands. Additionally, IL-1-stimulated NF$\kappa \mathrm{B}, \mathrm{JNK}$, and p38 expressions were rigorously faulty in cells deficient in IRAK4. LPS-induced stimulation of JNK was also repressed, and LPS-induced NF- $\kappa \mathrm{B}$ stimulation was postponed [33]. Furthermore, some studies documented that the kinase activity of IRAK4 was vital for its action. Using IRAK4-deficient murine embryonic fibroblasts that were reconstructed with an IRAK4 kinase inactive mutant, researchers indicated that the kinase activity of IRAK4 was necessary for the best stimulation of IL-1-induced NF- $\kappa \mathrm{B}$, JNK activation, and proinflammatory cytokines [34]. What is more, studies on IRAK4 kinase inactive knock-in mice showed that the kinase activity of IRAK4 is necessary for its action $[40,41]$. One study reported there are three phosphorylation sites (T342, T345, and S346) that are responsible for IRAK4 kinase activity [35]. As expected, our results indicated that LPS/D-Gal-stimulated IRAK4 activation demonstrated 


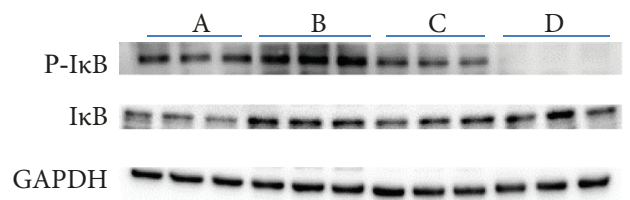

(a)

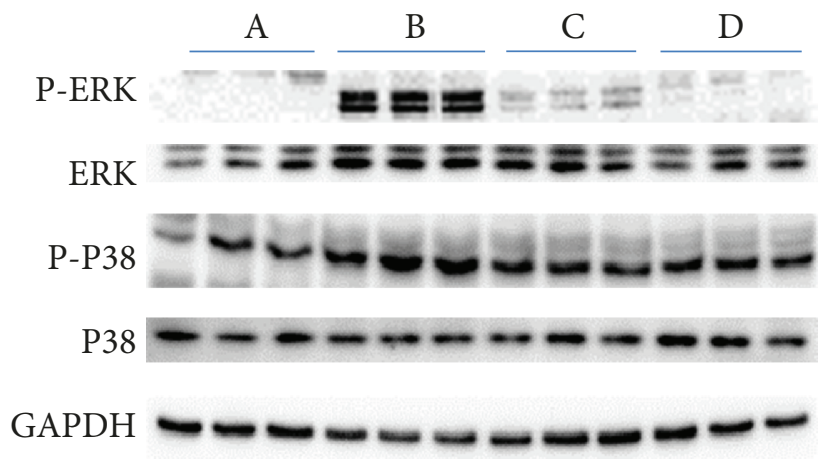

(c)

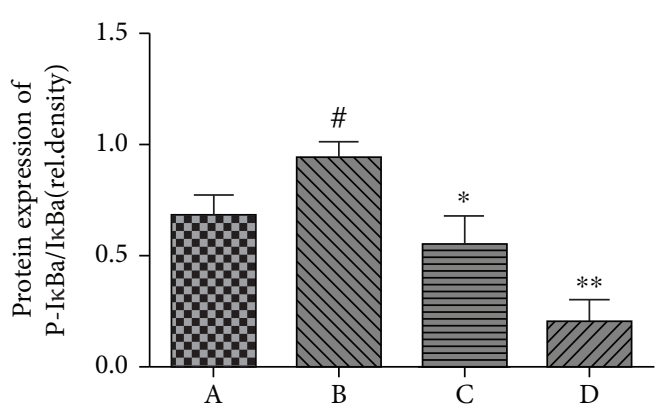

(b)
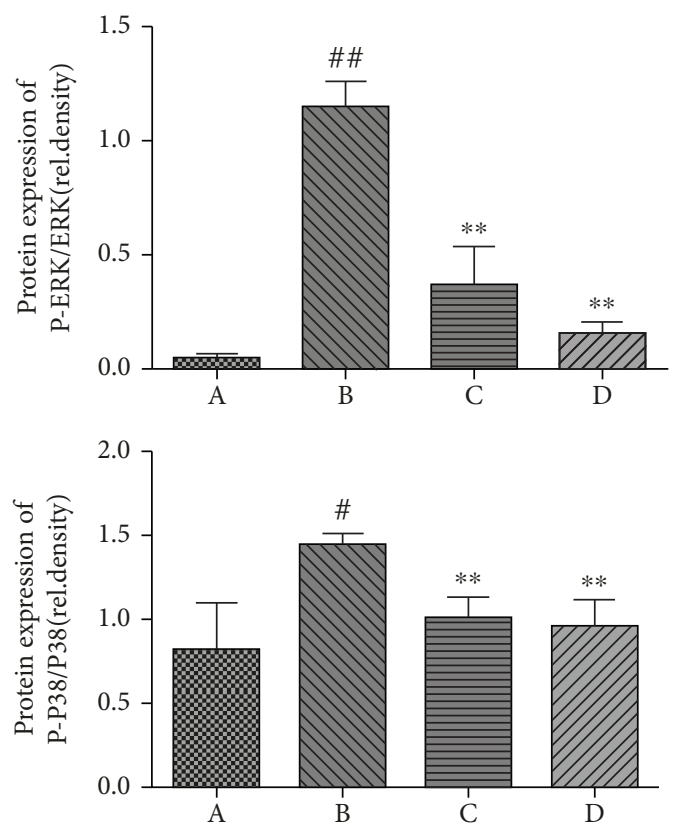

(d)

FIGURE 5: Participation of MAPK plus NF- $\kappa$ B signaling pathways in the anti-inflammatory effect of oridonin on LPS/D-Gal-induced ALI. (a) Level of P-I $\kappa \mathrm{B}$ was inhibited by oridonin in a concentration-dependent way, which was prompted by LPS/D-Gal challenge $(P<0.05)$. (b) Band intensity in western blot was quantified by ImageJ. (c) Compared to the control group, the phosphorylation levels of MAPK (ERK as well as P38) were suggestively elevated in the ALI group $(P<0.01)$. Pretreatment with oridonin significantly inhibited phosphorylation of MAPK $(P<0.01)$. (d) Band intensity in western blot was computed using ImageJ. ${ }^{\#} P<0.05,{ }^{\# \#} P<0.01$ vs. control group (A); ${ }^{*} P<0.05$, ${ }^{* *} P<0.01$ vs. model group (B).

by upregulation of P-IRAK4 (T345/S346), which was inhibited by oridonin treatment. However, macrophages from IRAK4 kinase inactive knock-in mice indicated that the kinase activity of IRAK4 was necessary for the stimulation of IL-1/TLR4-induced NF- $\kappa$ B [40, 41]. Moreover, there was obvious diminishing of IL-1/TLR-induced cytokine as well as chemokine generation in IRAK4 kinase inactive knockin mice [41-43]. Why are there two opposite conclusions? At present, different cell types are the causes of different results, and the specific mechanism has yet to be further studied. However, at least it is certain that IRAK4 kinase activity is necessary for the optimal inflammatory signaling pathway mediated by IL-1/TLR4.

All IRAK family members share analogous domain structures, comprising a conserved N-terminal death domain plus a central kinase domain [44]. IRAK4 binds to the adaptor protein MyD88 via its death domain. The downstream signaling pathway fails to activate without combination of the two proteins [45]. Thus, we infer that the death domain of IRAK4 has a crucial role in molecular recognition and activation of the downstream signaling pathway. Protein acetylation and the reverse process, named deacetylation, are PTMs. Histone acetyltransferases (HATs) as well as histone deacetylases (HDACs) could control gene expression by modifying histone proteins [46]. Nonetheless, HAT and HDAC can modulate precise signaling cascades and have additional targets apart from histones, comprising NF- $\kappa \mathrm{B}$, signal transducer, and activator of transcription 3 (STAT3) as well as p53 [47-50]. Scientists have identified 3600 lysine acetylation sites on 1750 proteins, and the modification preferentially targets large macromolecular complexes [51]. Their findings support the concept that acetylation might control innate immune response-related proteins, for example, DDX58, IRAK4 (K34), OAS2, and TRIM25 [51]. K34, the acetylation modification target of IRAK4, is located in the death domain of IRAK4. Our results suggested that 


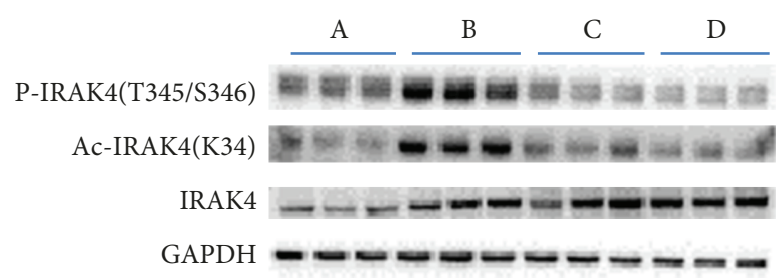

(a)
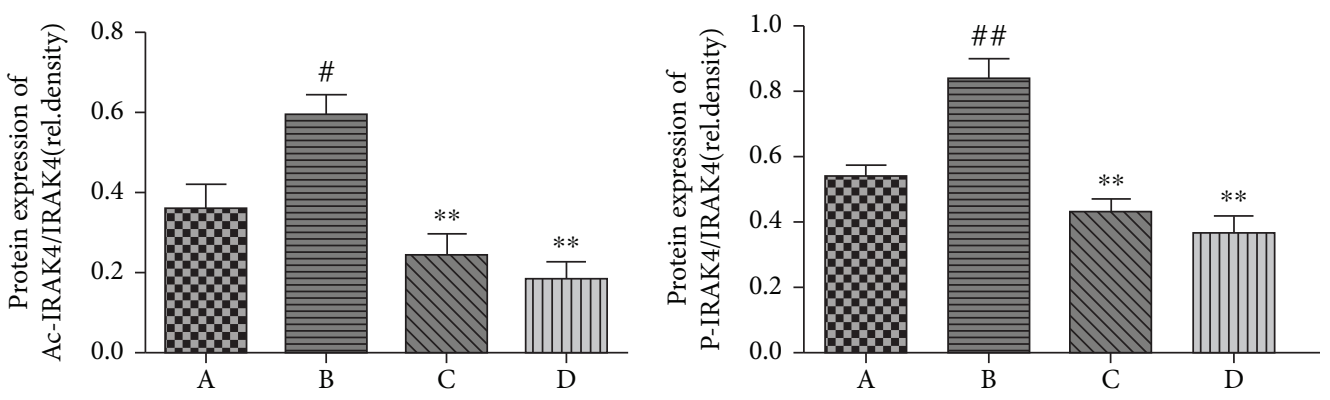

(b)

FIGURE 6: Modifications (phosphorylation and acetylation) of IRAK4 were impeded in LPS/D-Gal-induced ALI upon oridonin treatment. (a) Oridonin inhibited expression of P-IRAK4 (T345/S346) and acetyl-IRAK4 (K34) prompted by LPS/D-Gal. (b) Band intensity in western blot was quantified using Image J. ${ }^{\#} P<0.05,{ }^{\# \#} P<0.01$ vs. control group $(\mathrm{A}) ;{ }^{* *} P<0.01$ vs. model group (B).

LPS/D-Gal can upregulate the level of acetyl-IRAK4 (K34), and we speculated that the acetylation of IRAK4 (K34) may promote the binding of IRAK4 to MyD88, thus enabling activation of downstream MyD88-dependent signaling pathways. Our results showed that oridonin pretreatment with LPS/D-Gal-induced ALI inhibits acetylation of IRAK4, resulting in blockade of the TLR4 signaling pathway.

However, our study had certain limitations, which require further studies. First of all, our results that could not provide direct and sufficient evidence to support the acetylation status of IRAK4 (K34) may influence the combination of MyD88 and IRAK4. We need to construct the wild type, site-modified type, and mutant type of IRAK4 to further clarify the different binding capacities with MyD88. Second, we should further identify whether these two PTMs are cross-linked.

\section{Conclusions}

In conclusion, our study revealed that the protective effects of oridonin on LPS/D-Gal-induced ALI are mediated by inhibition of the PTMs of IRAK4, including phosphorylation of T345/S346 and acetylation of K34.

\section{Data Availability}

The data used to support the findings of this study are available from the corresponding author upon request.

\section{Disclosure}

Part of the study has been presented as conference abstract in Abstracts of the 26th Annual Conference of APASL [52].

\section{Conflicts of Interest}

The authors of this manuscript have no conflicts of interest to disclose.

\section{Authors' Contributions}

Min Shi and Yilin Deng contributed equally to this work and share first authorship.

\section{Acknowledgments}

This work was performed at the NPFPC Key Laboratory of Contraceptives and Devices, Shanghai Institute of Planned Parenthood Research (SIPPR), Institutes of Reproduction and Development, Fudan University, Shanghai, China. This work was supported/funded by the National Natural Science Foundation of China (Nos. 81570549, 81871912, and 81472242).

\section{Supplementary Materials}

See Supplementary Tables S1-S2 in the Supplementary Materials for comprehensive image analysis. Figure S1: A. $\mathrm{GO}$ analysis of target genes for biological processes revealed that the downregulated genes were highly concentrated in chemotaxis, locomotory behavior, and inflammatory response biological processes. B. KEGG pathway analysis of target genes showed that the downregulated genes are enriched in several pathways including the NOD-like receptor signaling pathway and chemokine signaling pathway. (Supplementary Materials)

\section{References}

[1] N. Kaplowitz, "Liver biology and pathobiology," Hepatology, vol. 43, no. S1, pp. S235-S238, 2006. 
[2] W. Tian and Y. Xu, "Decoding liver injury: a regulatory role for histone modifications," The International Journal of Biochemistry \& Cell Biology, vol. 67, pp. 188-193, 2015.

[3] S. K. Asrani, H. Devarbhavi, J. Eaton, and P. S. Kamath, "Burden of liver diseases in the world," Journal of Hepatology, vol. 70, no. 1, pp. 151-171, 2019.

[4] R. Ferriero, E. Nusco, R. de Cegli, A. Carissimo, G. Manco, and N. Brunetti-Pierri, "Pyruvate dehydrogenase complex and lactate dehydrogenase are targets for therapy of acute liver failure," Journal of Hepatology, vol. 69, no. 2, pp. 325-335, 2018.

[5] S. L. Planey, R. Kumar, and J. A. Arnott, "Post-translational modification of transcription factors: mechanisms and potential therapeutic interventions," Current Molecular Pharmacology, vol. 6, no. 3, pp. 173-182, 2013.

[6] A. S. Venne, L. Kollipara, and R. P. Zahedi, "The next level of complexity: crosstalk of posttranslational modifications," Proteomics, vol. 14, no. 4-5, pp. 513-524, 2014.

[7] K. Helin and D. Dhanak, "Chromatin proteins and modifications as drug targets," Nature, vol. 502, no. 7472, pp. 480-488, 2013.

[8] W. Yao, T. Wang, and F. Huang, "p300/CBP as a key nutritional sensor for hepatic energy homeostasis and liver fibrosis," BioMed Research International, vol. 2018, Article ID 8168791, 14 pages, 2018.

[9] N. A. Osna, W. G. Carter, M. Ganesan et al., “Aberrant posttranslational protein modifications in the pathogenesis of alcohol-induced liver injury," World Journal of Gastroenterology, vol. 22, no. 27, pp. 6192-6200, 2016.

[10] C. Ji, "Advances and new concepts in alcohol-induced organelle stress, unfolded protein responses and organ damage," Biomolecules, vol. 5, no. 2, pp. 1099-1121, 2015.

[11] K. Murata, M. Hamada, K. Sugimoto, and T. Nakano, "A novel mechanism for drug-induced liver failure: inhibition of histone acetylation by hydralazine derivatives," Journal of Hepatology, vol. 46, no. 2, pp. 322-329, 2007.

[12] T. Hitosugi and J. Chen, "Post-translational modifications and the Warburg effect," Oncogene, vol. 33, no. 34, pp. 4279-4285, 2014.

[13] H. Ü. Kaniskan, M. L. Martini, and J. Jin, "Inhibitors of protein methyltransferases and demethylases," Chemical Reviews, vol. 118, no. 3, pp. 989-1068, 2018.

[14] T. Lanyon-Hogg, M. Faronato, R. A. Serwa, and E. W. Tate, "Dynamic protein acylation: new substrates, mechanisms, and drug targets," Trends in Biochemical Sciences, vol. 42, no. 7, pp. 566-581, 2017.

[15] C. Y. Li, E. Q. Wang, Y. Cheng, and J. K. Bao, “Oridonin: an active diterpenoid targeting cell cycle arrest, apoptotic and autophagic pathways for cancer therapeutics," The International Journal of Biochemistry \& Cell Biology, vol. 43, no. 5, pp. 701-704, 2011.

[16] T. Zhen, C. F. Wu, P. Liu et al., "Targeting of AML1-ETO in $\mathrm{t}(8 ; 21)$ leukemia by oridonin generates a tumor suppressorlike protein," Science Translational Medicine, vol. 4, no. 127, article 127ra38, 2012.

[17] Y. Guo, Q. Shan, Y. Gong, J. Lin, X. Yang, and R. Zhou, "Oridonin in combination with imatinib exerts synergetic anti-leukemia effect in $\mathrm{Ph}+$ acute lymphoblastic leukemia cells in vitro by inhibiting activation of LYN/mTOR signaling pathway," Cancer Biology \& Therapy, vol. 13, no. 13, pp. 1244-1254, 2012.
[18] T. Ikezoe, Y. Yang, K. Bandobashi et al., "Oridonin, a diterpenoid purified from Rabdosia rubescens, inhibits the proliferation of cells from lymphoid malignancies in association with blockade of the NF- $\kappa \mathrm{B}$ signal pathways," Molecular Cancer Therapeutics, vol. 4, no. 4, pp. 578-586, 2005.

[19] G. Chen, K. Wang, B. Y. Yang, B. Tang, J.-X. Chen, and Z.-C. Hua, "Synergistic antitumor activity of oridonin and arsenic trioxide on hepatocellular carcinoma cells," International Journal of Oncology, vol. 40, no. 1, pp. 139-147, 2012.

[20] F. H. Gao, X. H. Hu, W. Li et al., "Oridonin induces apoptosis and senescence in colorectal cancer cells by increasing histone hyperacetylation and regulation of p16, p21, p27 and c-myc," BMC Cancer, vol. 10, no. 1, article 610, 2010.

[21] W. Guo, P. Zheng, J. Zhang, L. Ming, C. Zhou, and S. Zhang, "Oridonin suppresses transplant rejection by depleting $\mathrm{T}$ cells from the periphery," International Immunopharmacology, vol. 17, no. 4, pp. 1148-1154, 2013.

[22] S. Wang, H. Yang, L. Yu et al., "Oridonin attenuates $\mathrm{A} \beta_{1-42^{-}}$ induced neuroinflammation and inhibits NF- $\kappa \mathrm{B}$ pathway," PLoS One, vol. 9, no. 8, article e104745, 2014.

[23] Z. Y. Zhang, R. Daniels, and H. J. Schluesener, "Oridonin ameliorates neuropathological changes and behavioural deficits in a mouse model of cerebral amyloidosis," Journal of Cellular and Molecular Medicine, vol. 17, no. 12, pp. 1566-1576, 2013.

[24] F. J. Bohanon, X. Wang, B. M. Graham et al., "Enhanced effects of novel oridonin analog CYD0682 for hepatic fibrosis," The Journal of Surgical Research, vol. 199, no. 2, pp. 441-449, 2015.

[25] Y. Deng, C. Chen, H. Yu et al., "Oridonin ameliorates lipopolysaccharide/D-galactosamine-induced acute liver injury in mice via inhibition of apoptosis," American Journal of Translational Research, vol. 9, no. 9, pp. 4271-4279, 2017.

[26] M. Shi, X. J. Lu, J. Zhang et al., “Oridonin, a novel lysine acetyltransferases inhibitor, inhibits proliferation and induces apoptosis in gastric cancer cells through p53- and caspase-3mediated mechanisms," Oncotarget, vol. 7 , no. 16, pp. 22623-22631, 2016.

[27] H. Yu, H. Diao, C. Wang et al., "Acetylproteomic analysis reveals functional implications of lysine acetylation in human spermatozoa (sperm)," Molecular \& Cellular Proteomics, vol. 14, no. 4, pp. 1009-1023, 2015.

[28] G. L. Su, "Lipopolysaccharides in liver injury: molecular mechanisms of Kupffer cell activation," American Journal of Physiology. Gastrointestinal and Liver Physiology, vol. 283, no. 2, pp. G256-G265, 2002.

[29] Y. C. Lu, W. C. Yeh, and P. S. Ohashi, "LPS/TLR4 signal transduction pathway," Cytokine, vol. 42, no. 2, pp. 145-151, 2008.

[30] L. Chang and M. Karin, "Mammalian MAP kinase signalling cascades," Nature, vol. 410, no. 6824, pp. 37-40, 2001.

[31] M. S. Hayden and S. Ghosh, "Shared principles in NF- $\kappa$ B signaling," Cell, vol. 132, no. 3, pp. 344-362, 2008.

[32] J. Liu, C. Qian, and X. Cao, "Post-translational modification control of innate immunity," Immunity, vol. 45, no. 1, pp. 15-30, 2016.

[33] N. Suzuki, S. Suzuki, G. S. Duncan et al., "Severe impairment of interleukin-1 and Toll-like receptor signalling in mice lacking IRAK-4," Nature, vol. 416, no. 6882, pp. 750-754, 2002.

[34] E. Lye, C. Mirtsos, N. Suzuki, S. Suzuki, and W. C. Yeh, “The role of interleukin 1 receptor-associated kinase-4 (IRAK-4) kinase activity in IRAK-4-mediated signaling," Journal of Biological Chemistry, vol. 279, no. 39, pp. 40653-40658, 2004. 
[35] H. Cheng, T. Addona, H. Keshishian et al., "Regulation of IRAK-4 kinase activity via autophosphorylation within its activation loop," Biochemical and Biophysical Research Communications, vol. 352, no. 3, pp. 609-616, 2007.

[36] S. Flannery and A. G. Bowie, "The interleukin-1 receptorassociated kinases: critical regulators of innate immune signalling," Biochemical Pharmacology, vol. 80, no. 12, pp. 1981-1991, 2010.

[37] R. Silverstein, "D-galactosamine lethality model: scope and limitations," Journal of Endotoxin Research, vol. 10, no. 3, pp. 147-162, 2004.

[38] A. S. J. Baldwin Jr., "The NF- $\kappa$ B and $I \kappa$ B proteins: new discoveries and insights," Annual Review of Immunology, vol. 14, no. 1, pp. 649-681, 1996.

[39] T. P. Garrington and G. L. Johnson, "Organization and regulation of mitogen-activated protein kinase signaling pathways," Current Opinion in Cell Biology, vol. 11, no. 2, pp. 211-218, 1999.

[40] T. Kawagoe, S. Sato, A. Jung et al., "Essential role of IRAK-4 protein and its kinase activity in Toll-like receptor-mediated immune responses but not in TCR signaling," Journal of Experimental Medicine, vol. 204, no. 5, pp. 1013-1024, 2007.

[41] T. W. Kim, K. Staschke, K. Bulek et al., "A critical role for IRAK4 kinase activity in Toll-like receptor-mediated innate immunity," Journal of Experimental Medicine, vol. 204, no. 5, pp. 1025-1036, 2007.

[42] M. Koziczak-Holbro, C. Joyce, A. Glück et al., "IRAK-4 kinase activity is required for interleukin-1 (IL-1) receptor- and Tolllike receptor 7-mediated signaling and gene expression," Journal of Biological Chemistry, vol. 282, no. 18, pp. 1355213560, 2007.

[43] X. Li, "IRAK4 in TLR/IL-1R signaling: possible clinical applications," European Journal of Immunology, vol. 38, no. 3, pp. 614-618, 2008.

[44] Z. Wang, J. Liu, A. Sudom et al., "Crystal structures of IRAK-4 kinase in complex with inhibitors: a serine/threonine kinase with tyrosine as a gatekeeper," Structure, vol. 14, no. 12, pp. 1835-1844, 2006.

[45] A. Kuglstatter, A. G. Villaseñor, D. Shaw et al., "Cutting edge: IL-1 receptor-associated kinase 4 structures reveal novel features and multiple conformations," The Journal of Immunology, vol. 178, no. 5, pp. 2641-2645, 2007.

[46] H. A. Doyle and M. J. Mamula, "Autoantigenesis: the evolution of protein modifications in autoimmune disease," Current Opinion in Immunology, vol. 24, no. 1, pp. 112-118, 2012.

[47] J. Y. Lim, M. A. Oh, W. H. Kim, H. Y. Sohn, and S. I. Park, "AMP-activated protein kinase inhibits TGF- $\beta$-induced fibrogenic responses of hepatic stellate cells by targeting transcriptional coactivator p300," Journal of Cellular Physiology, vol. 227, no. 3, pp. 1081-1089, 2012.

[48] M. Breaux, K. Lewis, L. Valanejad et al., "p300 regulates liver functions by controlling p53 and C/EBP family proteins through multiple signaling pathways," Molecular and Cellular Biology, vol. 35, no. 17, pp. 3005-3016, 2015.

[49] Z. L. Yuan, Y. J. Guan, D. Chatterjee, and Y. E. Chin, "Stat3 dimerization regulated by reversible acetylation of a single lysine residue," Science, vol. 307, no. 5707, pp. 269-273, 2005.

[50] L. Chen, W. Fischle, E. Verdin, and W. C. Greene, "Duration of nuclear NF- $\kappa$ B action regulated by reversible acetylation," Science, vol. 293, no. 5535, pp. 1653-1657, 2001.
[51] C. Choudhary, C. Kumar, F. Gnad et al., "Lysine acetylation targets protein complexes and co-regulates major cellular functions," Science, vol. 325, no. 5942, pp. 834-840, 2009.

[52] Y. Deng, G. Li, and C. Shi, "Abstracts of the 26th Annual Conference of APASL, February 15-19, 2017, Shanghai, China," Hepatology International, vol. 11, Supplement 1, pp. 1-1093, 2017. 


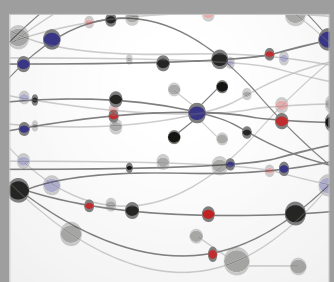

The Scientific World Journal
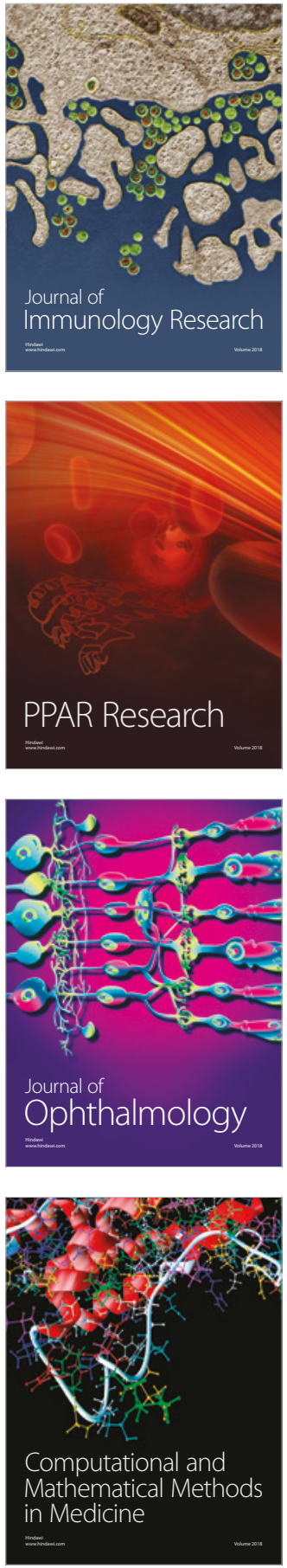

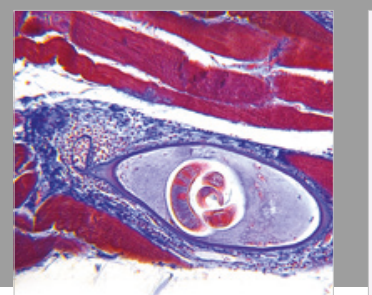

Gastroenterology Research and Practice

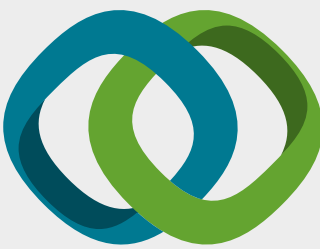

\section{Hindawi}

Submit your manuscripts at

www.hindawi.com
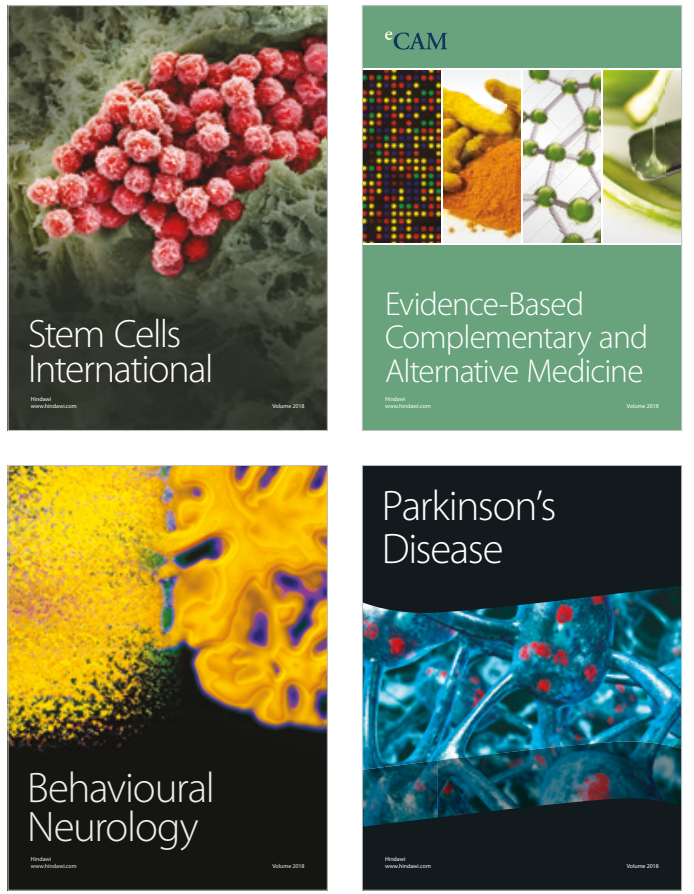

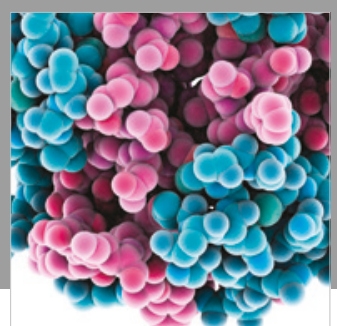

ournal of

Diabetes Research

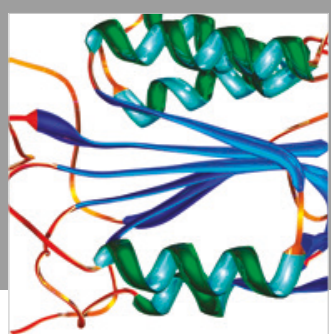

Disease Markers
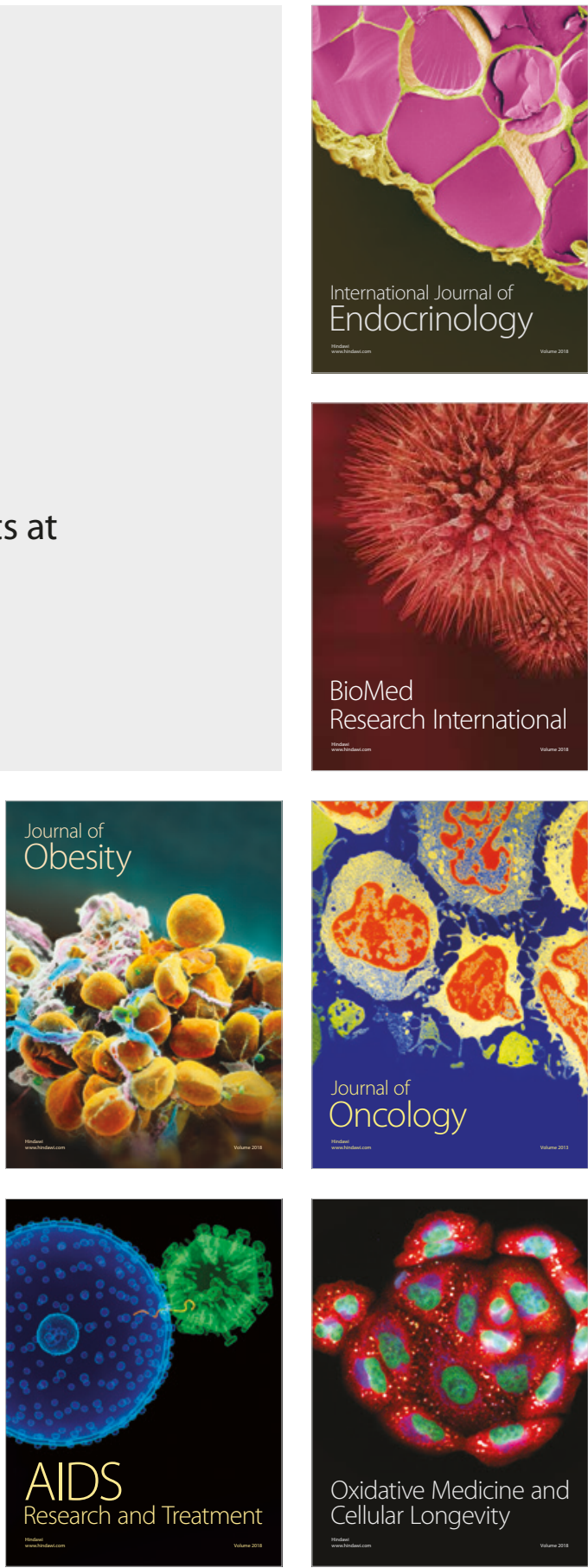